\title{
Recent advances in the treatment of premature ejaculation
}

This article was published in the following Dove Press journal:

Drug Design, Development and Therapy

6 January 2010

Number of times this article has been viewed

\section{Kate D Linton \\ Kevan R Wylie}

Sheffield Teaching Hospitals, Sheffield, UK
Correspondence: Kevan R Wylie

Royal Hallamshire Hospital,

Glossop Road,

Sheffield SIO 2JF, UK

Tel +44 I I 427 I 3334

Fax +44 II 4 27I 8693

Email k.r.wylie@sheffield.ac.uk

\begin{abstract}
Premature ejaculation (PE) is the most common sexual problem affecting men. It can affect men at all ages and has a serious impact on the quality of life for men and their partners. Currently there are no pharmaceutical agents approved for use in the UK, and so all drugs used for this condition are off label. Behavioral therapy has been used to treat PE, but the results are not durable once therapy has been concluded. Several topical therapies have been used including severance-secret (SS) cream, lignocaine spray, lidocaine-prilocaine cream and lidocaine-prilocaine spray (TEMPE). There has been recent interest in the selective serotonin reuptake inhibitors (SSRIs) for the treatment of PE, due to the fact that one of their common side effects is delayed ejaculation. Currently used SSRIs have several non-sexual side effects and long half lives, therefore there has been interest in developing a short acting, efficacious SSRI that can be used on-demand for PE. Dapoxetine has been recently evaluated for the treatment of PE by several groups, and results so far appear promising.
\end{abstract}

Keywords: premature ejaculation, topical therapies, selective serotonin re-uptake inhibitor, dapoxetine

\section{Introduction}

Premature ejaculation (PE), early or rapid ejaculation is the most common sexual disorder affecting men. A national study of sexual attitudes and lifestyles found PE lasting 1 month in the previous year to have a prevalence of $11.7 \%$ and 6 months in the previous year had a prevalence of $2.9 \% .{ }^{1}$ Unlike erectile dysfunction (ED), PE affects men of all ages equally but like ED can have a serious impact on quality of life for both the patient and his partner. It may be lifelong, present since the onset of sexual maturity, or can be acquired after previously normal ejaculation, for example after a urological or psychological event. It has been suggested that there may be other forms of PE, including premature-like ejaculatory dysfunction where men with normal ejaculatory latency times perceive their ejaculation to be premature, and natural variable PE which might occur in a specific situation. ${ }^{2}$ The diagnosis of PE is evolving, however, one recommended definition of PE is persistent or recurrent ejaculation that occurs with minimal sexual stimulation before, on or shortly after penetration and before the person wishes it to; and over which the sufferer has no voluntary control, which causes marked distress or interpersonal difficulties. It cannot be due to the direct effects of a substance (Diagnostic and Statistical Manual of Mental Disorders Fourth Edition, text revision, [DSM-IV-TR]). The International Society for Sexual Medicine (ISSM) definition states that PE is "A male sexual dysfunction characterized by ejaculation which is always or nearly always occurs prior submit your manuscript | www.dovepress.com

Dovepress
Drug Design, Development and Therapy 2010:4 I-6

(C) 2010 Linton and Wylie, publisher and licensee Dove Medical Press Ltd. This is an Open Access article which permits unrestricted noncommercial use, provided the original work is properly cited. 
to or within 1 minute of vaginal penetration; and an inability to delay ejaculation on all or nearly all vaginal penetrations, and negative personal consequences, such as distress, bother, frustration, and/or the avoidance of sexual intimacy." ${ }^{3}$ In practice the intra-vaginal ejaculatory latency time (IELT) is often used as a method of quantifying the response to treatment and as a standardized method of comparing treatments within clinical trials. The IELT is defined as the time from vaginal intromission to intravaginal ejaculation. ${ }^{4}$

Until relatively recently $\mathrm{PE}$ was treated mainly by behavioral techniques, eg, the "squeeze technique" which was first described by Masters and Johnson in $1970,{ }^{5}$ and the "stop-start method" described by Semans in $1956 .{ }^{6}$ Despite the high prevalence of this condition, there is little research regarding its causation. It is likely that both biological and psychological factors have a role to play. Penile hypersensitivity, hyperexcitable ejaculatory reflex, increased sexual arousability, possible endocrinopathy; genetic predisposition and 5-hydroxy-tryptamine (5-HT) receptor dysfunction have all been implicated as being biological causative factors. ${ }^{7}$ Several psychological risk factors have been implicated including anxiety, social phobia, relationship problems, infrequent sexual intercourse and lack of sexual experience. ${ }^{8}$

At the present time there are no pharmacological agents approved in the UK for use in PE and all drugs are therefore used "off-label". There are several treatment options available to men with PE. These include behavioral therapy, topical therapies, and systemic pharmacological agents.

\section{Behavioral therapies}

Behavioral therapies include the 'stop-start' and 'squeeze technique'; these require commitment from the man and his partner, as well as the availability and instruction from well trained sex therapists. The stop-start technique involves the man stimulating himself to the point just before ejaculation, and then stopping. Once the sensations have subsided, he starts again. This should be repeated three times. The length of time before each stop gets gradually longer. The squeeze technique involves the partner (or man) using their fingers to squeeze the head (glans) of the penis to cause the erection (and ejaculation) to subside. Unfortunately most men do not show any lasting improvement using either of these techniques. ${ }^{9}$

\section{Pharmacological treatment Topical agents}

The theory that men with PE may have penile hypersensitivity provides a rationale for using topical agents for example topical desensitizing agents. The use of local anesthetic treatment to delay ejaculation was first described by Schapiro in $1943 .{ }^{10}$ Topical agents are very attractive as they can be used on an as needed basis and systemic side effects are likely to be minimal. They are reported to be very efficacious, however studies are often small and there are often local side effects.

\section{Severance-secret (SS) cream}

SS cream (Cheil Jedan Corporation, Seoul, Korea) is a mixture of nine traditional medicines, including Korean ginseng, bufonoid venom and cinnamon. Some of these have local anesthetic as well as vasoactive properties. In a randomized, doubleblind, placebo-controlled trial of SS-cream versus placebo, there was an increase in mean IELT from 1.37 minutes before treatment to 10.92 minutes in the SS-cream group. ${ }^{11} \mathrm{SS}$ cream is available for use only in Korea and all of the studies evaluating its efficacy have been performed there and by the same research group. It has to be used an hour before intercourse and washed off immediately before intercourse, and some patients complain that it has an unpleasant smell and color. Subsequently a renewed SS cream has been formulated that has the two main components of the SS cream, namely Korean ginseng and bufonoid venom in a hydrobase with an enhancer, yet without the smell and color of the original SS cream. ${ }^{12} \mathrm{SS}$ cream is not approved for use in Europe or the USA.

\section{Lignocaine spray}

Marketed as Stud 100 or Premjact, this spray has been available for many years, and can be bought over the counter without a prescription. The active ingredient within the spray is the local anesthetic lignocaine $(9.6 \%)$. Theoretically this spray would work in the same way as other topical anesthetic agents, however there is a paucity of data from clinical trials to support its use.

\section{Lidocaine-prilocaine cream}

The eutectic mixture of local anesthetic (EMLA ${ }^{\mathrm{TM}}$; AstraZeneca, London, UK) is a local anesthetic cream that contains $2.5 \%$ of both lidocaine and prilocaine for topical application. Trials using this topically in men with PE are small. In a trial of 42 men of whom only 29 completed the study, the IELT increased from 1.49 minutes to 8.45 minutes following 2 months use of this local anesthetic cream. However genital hypoesthesia has been reported in both sexes in this study. ${ }^{13}$

\section{Lidocaine-prilocaine spray}

Topical eutectic mixture for premature ejaculation (TEMPE Plethora Solutions PLC, London, UK) is a formulation of 
lignocaine and prilocaine in a metered dose aerosol-delivery system. Each spray delivers $7.5 \mathrm{mg}$ of lidocaine and $2.5 \mathrm{mg}$ of prilocaine. It is fast acting and appears to be efficacious in small studies. It does not penetrate keratinized epithelium, and so only anesthetizes the glans; however there still appears to be some hypoesthesia associated with its use. ${ }^{14}$ In a phase II trial of 56 patients, TEMPE increased the IELT $2.4 \times$ higher than placebo. ${ }^{14}$ In a larger multicenter phase III trial of 300 patients, TEMPE increased the IELT from 0.6 minutes to 3.8 minutes and was well tolerated by patients and partners as well as being rated good or excellent by $66 \%$ of patients using the treatment. ${ }^{15}$ Although hypoesthesia has not been a reported problem in the female partners, there has been a small number of women who have reported a burning sensation during intercourse. ${ }^{14,15}$

\section{Dyclonine/alprostadil}

Dyclonine is a local anesthetic usually used in the field of dentistry. It has been combined with the vasodilator alprostadil, and used to treat PE. The product is applied to the tip of the penis in the region of the meatus. One pilot study claims positive results with it, however the data is limited and further studies are warranted before conclusions regarding this combination can be drawn. ${ }^{16}$

\section{Systemic therapies \\ Tramadol}

The oral opioid analgesic tramadol (Ultram ${ }^{\circledR}$; Johnson \& Johnson, NJ, USA) has been used as a treatment for PE. It is a centrally acting analgesic with two mechanisms of action. It exerts an effect on the $\mu$-opioid receptor, but also inhibits noradrenaline and serotonin reuptake. Its mechanism of action in PE is poorly understood, however it is thought to be related to its action on the $\mu$-opioid receptor, which may reduce sensitivity, as well as the inhibition of serotonin reuptake, which may delay ejaculation. There have been two small clinical trials and both showed a significant increase in the IELT compared with placebo. ${ }^{17,18} \mathrm{~A}$ study of 60 men comparing $25 \mathrm{mg}$ of tramadol to placebo in a single blind cross-over study showed that tramadol increased the IELT from 1.17 minutes to 7.37 minutes. Tramadol increased patients' control of ejaculation as well their sexual satisfaction. ${ }^{17}$ A further study of 64 men comparing $50 \mathrm{mg}$ of tramadol with placebo showed an increase in IELT from 19 seconds to over 4 minutes in the tramadol arm. ${ }^{18}$ Although results from these studies are encouraging, further studies are warranted.

\section{Clomipramine}

Clomipramine (Anafranil ${ }^{\mathrm{TM}}$; Mallinckrodt pharmaceutical Products, MS, USA) is a tricyclic antidepressant that inhibits the reuptake of noradrenaline and serotonin. It is commonly used in the treatment of obsessive-compulsive disorders. Studies have used continuous dosing as well as on-demand dosing and shown significant increases in the IELT. ${ }^{19,20}$ A meta-analysis evaluating the systemic treatments for PE, found clomipramine to be efficacious, particularly continuous dosing, results also showed it to be comparable to the selective serotonin re-uptake inhibitors (SSRIs) in its effects. ${ }^{19}$ On demand clomipramine has been shown to increase the IELT 4-fold, however patients did have a high incidence of side effects. ${ }^{20}$

\section{Serotonergic antidepressants}

Psychotropic medications, for example SSRIs, have been used to delay ejaculation, and in fact can prolong intra-vaginal ejaculatory latency time for several minutes. They are usually used for the treatment of depression, and it was noted that delayed ejaculation was one of their common side-effects. They are often used at lower doses than when used to treat depression. Side effects include dry mouth, drowsiness, nausea and reduced libido as well as erectile dysfunction ${ }^{21}$. Currently four SSRIs are commonly used in the treatment of PE, Fluoxetine (Prozac ${ }^{\circledR}$; Eli Lilly, IN, USA), paroxetine (Paxil ${ }^{\circledR}$; GlaxoSmithKline PLC, PA, USA), sertraline $\left(\right.$ Zoloft $^{\circledR}$; Pfizer, NY, USA) and citalopram (Celexa ${ }^{\circledR}$; Forest Laboratories, NY, USA).

At least three serotonin receptor subtypes have been identified as having a role in ejaculation, these include $5-\mathrm{HT}_{1 \mathrm{a}}$, $5-\mathrm{HT}_{1 \mathrm{~b}}$ and $5-\mathrm{HT}_{2 \mathrm{c}}$. Activation of $5-\mathrm{HT}_{1 \mathrm{a}}$ receptor has a pro-ejaculatory effect, however activation of the $5-\mathrm{HT}_{1 \mathrm{~b}}$ and $5-\mathrm{HT}_{2 \mathrm{c}}$ delays ejaculation. ${ }^{21}$ To prevent overstimulation of post-synaptic 5-HT receptors, 5-HT transporters immediately remove the 5-HT from the synapse back into the pre-synaptic neurone. ${ }^{22}$

Serotonin is released from the pre-synaptic neurons into the synapse and it activates the $5-\mathrm{HT}_{1 \mathrm{~B}}$ receptors. This results in reduced release of serotonin in the synapse. The SSRI's block 5-HT transporter mechanisms and so increase 5-HT within the synapses. This in turn activates $5-\mathrm{HT}_{1 \mathrm{~A}}$ and $5-\mathrm{HT}_{1 \mathrm{~B}}$ receptors resulting in inhibition of serotonin release into the synaptic cleft. The result is sustained mild stimulation of all post-synaptic 5-HT receptors. After several days to weeks of SSRI treatment, the receptors become desensitized and so there is a reduction of that inhibitory action they have on serotonin release. The overall effect is more serotonin 
released into the synapse. ${ }^{8}$ Activation of $5-\mathrm{HT}_{2 \mathrm{C}}$ receptors adjusts the ejaculatory set point and delays ejaculation, however the extent of this delay depends on several factors including the type, dose and frequency of the SSRI administration as well as the genetically determined ejaculatory threshold set point. ${ }^{23}$

Paroxetine, sertraline, and fluoxetine have all been evaluated in patients with PE, paroxetine however has been found to have substantially greater efficacy, followed by sertraline and fluoxetine. ${ }^{19}$ Unfortunately these drugs have other sexual side effects, reduced libido, anorgasmia and erectile dysfunction have been observed with all of those drugs. ${ }^{21}$ The other nonsexual side effects of SSRIs include insomnia, fatigue, nausea, constipation and loss of appetite. Abrupt cessation or reduction of SSRI therapy can be associated with a discontinuation syndrome. Symptoms include dizziness, nausea or vomiting, fatigue, headaches, ataxia, lethargy, anxiety, agitation and insomnia. Symptoms begin from 24 to 72 hours after discontinuance and may last more than a week. It is generally recommended that SSRIs should not be stopped suddenly but reduced over several weeks. ${ }^{23}$ This is thought to be more common with the SSRIs that have shorter half lives. ${ }^{21}$

The side effect profile of the SSRIss taken on a chronic basis has led to the suggestion that an "on-demand" SSRI may be useful for PE. Waldinger and colleagues have evaluated this using $25 \mathrm{mg}$ clomipramine and $20 \mathrm{mg}$ paroxetine. Although there was a clear benefit with clomipramine, paroxetine on the other hand did not show a significant increase in IELT when used in this manner. ${ }^{20}$ Abdel-Hamid and colleagues evaluated the effects of SSRIs on an as needed basis and found that paroxetine was superior to the pause-squeeze method, however only by a modest increase in the median IELT, 3 minutes for the squeeze technique and 4 minutes for paroxetine from a baseline of 1 minute. ${ }^{24}$

It has been postulated that on-demand treatment for PE with the conventional SSRIs would only be successful when combined with a 5-HT $\mathrm{HA}_{1 \mathrm{~A}}$ receptor antagonist or some other treatment that would acutely stimulate serotonin release. ${ }^{4}$ $5-\mathrm{HT}_{1 \mathrm{~A}}$ receptor antagonists in the pre-clinical setting have been shown to delay ejaculation when used concomitantly and acutely with an SSRI, but were not effective on delaying ejaculation when used alone. ${ }^{16}$ This is very promising, however further studies are required before conclusions can be drawn regarding this combination.

Although SSRIs do have a role in the treatment of PE, they must be used with caution and patients must be warned that there is a risk of suicidal ideation associated with the use of this class of drugs. Patients must be followed up and documentation that they are aware that their use is "off-label" and of the small but relevant suicide risk.

The need for an on-demand efficacious medication for the treatment of PE has led to the development of dapoxetine for this specific use.

\section{Dapoxetine}

Dapoxetine (Priligy ${ }^{\mathrm{TM}}$ Johnson \& Johnson, NJ, USA) is a novel SSRI, it is similar to the other SSRIs in that it exerts its effects through the inhibition of the serotonin reuptake transporter. ${ }^{21}$ In pharmacological studies it has been shown to be a highly potent inhibitor of the transporter. ${ }^{25}$ Dapoxetine is a short acting SSRI and so is probably better suited as an "on-demand" treatment for PE. There have been several randomized placebo controlled trials evaluating dapoxetine. Doses of 30 and $60 \mathrm{mg}$ have been used and peak plasma concentrations observed 1.01 and 1.27 hours after administration. Elimination is also rapid, the half life is 1.3 to 1.4 hours, and there appears to be very little accumulation. ${ }^{26}$ This is in contrast to the other SSRI's that have half lives in the order of 1 to 4 days and chronic use results in significant accumulation.

In several studies dapoxetine has been shown to significantly improve the intra-vaginal ejaculatory latency time as compared with baseline and placebo, IELT 1.66 minutes, 3.03 minutes and 3.15 minutes with placebo, dapoxetine $30 \mathrm{mg}$ and $60 \mathrm{mg}$ respectively, when the drug was taken 30 to 60 minutes before intercourse. When taken 3 to 4 hours prior to intercourse the IELT was 1.79 minutes, 3.06 minutes and 3.97 minutes with placebo, $30 \mathrm{mg}$ and $60 \mathrm{mg}$ dapoxetine respectively. ${ }^{27}$ Dapoxetine was shown to not only increase the IELT significantly, compared with placebo, but also to be effective from the first dose when taken 1 to 3 hours before intercourse. A further sub-analysis of the data this trial showed that men with a perceived greater control over their ejaculation also had prolonged IELT, and were more likely to be in the dapoxetine arms of the trial. ${ }^{28}$ A phase III placebo controlled trial using dapoxetine $60 \mathrm{mg}$, showed significant improvements in patient reported outcome measures over a 9-week period. ${ }^{29}$

A more recent phase III trial from 22 countries, evaluating dapoxetine $30 \mathrm{mg}$ and $60 \mathrm{mg}$ versus placebo showed that IELT was significantly increased with dapoxetine. At the end of 24 weeks the IELT had increased from 0.9 minutes to 1.9 minutes, 3.1 minutes, 3.5 minutes with placebo, dapoxetine $30 \mathrm{mg}$ and $60 \mathrm{mg}$. All patients reported outcome measures were significantly improved with dapoxetine versus placebo. ${ }^{30}$ Currently dapoxetine has not been approved for 
use in men with PE in the UK, it has been approved in several other European countries, however there appears to be evidence that it is efficacious and well tolerated.

\section{PDE-5 inhibitors}

Many men with PE, also have concomitant erectile dysfunction (ED). Whether the man with PE develops secondary ED due to anxiety regarding his $\mathrm{PE}$, or whether the man with ED ejaculates early during intercourse before his erection fails is unknown. There was however a rationale to use PDE-5 inhibitors in these men, which lead to the hypothesis that they may be efficacious in primary PE. A study comparing the effectiveness of clomipramine, sertraline, paroxetine, sildenafil and pause-squeeze technique in men found that there was a 15-fold increase in the IELT for the sildenafil group compared with a 3- to 4-fold increase with the other treatments, sildenafil was superior in terms of patient satisfaction also. ${ }^{24} \mathrm{~A}$ further study comparing sildenafil with paroxetine and the squeeze technique in patients with PE but no ED, again showed increased IELT in the sildenafil group compared to the other two treatment groups. ${ }^{31}$ Unfortunately further studies have failed to show a significant increase in IELT with sildenafil, however recent small studies using vardenafil compared to placebo ${ }^{32}$ or sertraline in a randomized, prospective, crossover study ${ }^{33}$ in men with lifelong PE, showed a significant increase in IELT with vardenafil as well as improvements in other patient reported outcome measures. Two small studies have shown that the combination of paroxetine and sildenafil has significant improvements in IELT compared to paroxetine alone, ${ }^{34,35}$ unfortunately there was a higher incidence of side effects with the combined treatment. Clearly further large studies using PDE-5 inhibitors are warranted.

\section{Experimental treatment options}

Wise and Watson suggested a novel device based on the penile hypersensitivity hypothesis. A de-sensitizing band had a pronounced effect on the intra-vaginal ejaculatory latency period; the ring was used on a daily basis for 30 minutes, for 6 weeks. Once it was worn the man was told that he would need to masturbate at least three times per week either alone or with a partner for about five minutes, or until he was almost at the "point of no return", this should be repeated twice and on the third time he should masturbate to ejaculation. This device is no longer available for patient use for economic reasons of viability of sales. It may become available again as more men present to clinicians for help with this condition. ${ }^{36}$
A surgical procedure consisting of a dorsal neurectomy with or without glanular augmentation with hyaluronic acid gel has been reported. Although there are reported positive results with significantly increased IELT, the two groups that underwent dorsal neurectomy or dorsal neurectomy and glandular augmentation, both had significant side effects, including penile numbness, paresthesia and pain. The group that underwent hyaluronic acid augmentation alone reported no adverse side effects. ${ }^{37}$ Dorsal neurectomy is not in widespread practice, however there is now data from five years study on the glans augmentation arm of the trial and the hyaluronic acid implants were well maintained, showing long-term efficacy for the treatment of premature ejaculation. ${ }^{38}$

Pulsed radiofrequency neuromodulation has been used to treat PE by the desensitization of the dorsal penile nerves. This was a small study consisting of 15 patients with primary PE. Initial results of this pilot study showed a significant increase in the IELT compared to baseline; there were no reported problems with pain, penile hypoesthesia, or erectile dysfunction post-procedure. ${ }^{39}$

\section{Conclusion}

Premature ejaculation is a widespread condition affecting men of all ages. It has significant effects on quality of life for both the man and his partner, therefore it is an important sexual problem. Currently there are no licensed medications used for PE, in the UK. Several pharmacological approaches have been used in its treatment, including using topical anesthetic agents, the opioid analgesic tramadol, SSRIs, as well as PDE-5 inhibitors. Dapoxetine is a short-acting SSRI that has been formulated specifically for the treatment of PE, and results so far look very promising.

\section{Disclosures}

Kevan R Wylie has been a consultant to both Janssen-Cilag and Plethora.

\section{References}

1. Mercer $\mathrm{CH}$, Fenton KA, Johnson AM, et al. Sexual function problems and help seeking behaviour in Britain: national probability sample survey. BMJ. 2003;327(7412):426-427.

2. Waldinger MD. Premature ejaculation: definition and drug treatment. Drugs. 2007;67:547-568.

3. McMahon CG, Althof S, Waldinger MD, et al. An evidence-based definition of lifelong premature ejaculation: report of the International Society of Sexual Medicine ad hoc committee for the definition of premature ejaculation. $B J U$ Int. 2008;102(3):338-350.

4. Waldinger MD, Quinn P, Dilleen M, Mundayat R, Schweitzer DH, Boolell M. A multinational population survey of intravaginal ejaculation latency time. J Sex Med. 2005;2(4):492-497.

5. Masters W, Johnson V. Human Sexual Inadequacy. Boston, MA: Little Brown and Company; 1970. 
6. Semans J. Premature ejaculation; a new approach. South Med J. 1956;49:353-358.

7. McMahon C. Premature ejaculation: past, present and future perspectives. J Sex Med. 2005;2(Suppl 2):94-95.

8. Wylie KR, Ralph D. Premature ejaculation: the current literature. Curr Opin Urol. 2005;15:393-398.

9. De Amicus LA, Goldberg DC, Lo Piccolo J, Friedman J, Davies L. Clinical follow-up of couples treated for sexual dysfunction. Arch Sex Behav. 1985; 14:467-489.

10. Schapiro B. Premature ejaculation, a review of 1130 cases. J Urol. 1943;50:374.

11. Choi HK, Jung GW, Moon KH, et al. Clinical study of SS-cream in patients with lifelong premature ejaculation. Urology. 2000;55: 257-261.

12. Tian L, Xin ZC, Xin H, et al. Effect of renewed SS-cream on spinal somatosensory evoked potential in rabbits. Asian J Androl. 2004;6: $15-18$.

13. Busato W, Galindo CC. Topical anesthetic use for treating premature ejaculation: a double blind, randomized, placebo-controlled study. B J U Int. 2004;93:1018-1021.

14. Dinsmore WW, Hackett G, Goldmeier D, et al. Topical eutectic mixture for premature ejaculation (TEMPE): a novel aerosol-delivery form of lidocaine-prilocaine for treating premature ejaculation. $B J U$ Int. 2006;99:369-375.

15. Dinsmore WW, Wyllie MG. PSD502 improves ejaculatory latency, control and sexual satisfaction when applied topically $5 \mathrm{~min}$ before intercourse in men with premature ejaculation: results of a phase III, multicentre, double-blind, placebo controlled study. B J U Int. 2009;103: 940-949.

16. Gurkan L, Oommen M, Hellstrom WJG. Premature ejaculation: current and future treatments. Asian J Androl. 2008;10(1):102-109.

17. Salem EA, Wilson SK, Bissada NK, Delk JR, Hellstrom WJ, Cleves MA. Tramadol $\mathrm{HCl}$ has promise in an on-demand use to treat premature ejaculation. $J$ Sex Med. 2008;5(1):188-193.

18. Safarinejad MR, Hosseini SY. Safety and efficacy of tramadol in the treatment of premature ejaculation: a double-blind, placebo controlled, fixed dose, randomized study. J Clin Psychopharmacol. 2006;26(1): 27-31.

19. Waldinger MD, Zwinderman AH, Schweitzer DH, Olivier B. Relevance of methodological design for the interpretation of efficacy of drug treatment of premature ejaculation: a systematic review and meta-analysis. Int J Impot Res. 2004;16:369-381.

20. Waldinger MD, Zwinderman AH, Olivier B. On-demand treatment of premature ejaculation with clomipramine and paroxetine: A randomized, double-blind fixed-dose study with stop-watch assessment. Eur Urol. 2004:46:510-515.

21. Hellstrom WJG. Emerging treatments for premature ejaculation: focus on dapoxetine. Neuropsychiatr Dis Treat. 2009;5:37-46.

22. Giuliano F, Clement P. Serotonin and premature ejaculation: from physiology to patient management. Eur Urol. 2006;50:454-466.

23. Sadeghi-Nejad H, Watson W. Premature ejaculation: current medical treatment and new directions. J Sex Med. 2008;5:1037-1050.

24. Abdel-Hamid IA, El Naggar EA, El Ginany AH. Assessment of as needed use of pharmacotherapy and the pause-squeeze technique in premature ejaculation. Int Impot Res. 2001;13:41-45.
25. Andersson KE, Mulhall JP, Wyllie MG. Pharmacokinetic and pharmacodynamic features of dapoxetine, a novel drug for "on demand" treatment of premature ejaculation. $B J U$ Int. 2006;97:311-315.

26. Modi NB, Dresser MJ, Simon M, Lin D, Desai D, Gupta S. Single and multiple dose pharmacokinetics of dapoxetine hydrochloride, a novel agent for the treatment of premature ejaculation. J Clin Pharmacol. 2006;46(3):301-309.

27. Pryor JL, Althof SE, Steidle C, et al. Efficacy and tolerability of dapoxetine in treatment of premature ejaculation: an integrated analysis of two double-blind, randomized-controlled trials. Lancet. 2006;368(9539):929-937.

28. Shabsigh R, Patrick DL, Rowland DL, Bull SA, Tesfaye F, Rothman M. Perceived control over ejaculation is central to treatment benefit in men with premature ejaculation: results from phase III trials with dapoxetine. B J U Int. 2008;102:824-828.

29. Kauffman JM, Rosen RC, Mudumbi RV, Tesfaye, F, Hashmonay R, Rivas D. Treatment benefit of dapoxetine for premature ejaculation: results from s placebo-controlled phase III trial. $B J U$ Int. 2008;103: 651-658.

30. Buvat J, Tesfaye F, Rothman M, Rivas DA, Giuliano F. Dapoxetine for the treatment of premature ejaculation: results from a randomized, double blind, placebo-controlled phase three trial in 22 countries. Eur Urol. 2009;55:957-968.

31. Wang WF, Wang Y, Minhas S, Ralph DJ. Can sildenafil treat primary premature ejaculation? A prospective clinical study. Int J Urol. 2007; 14:331-335

32. Aversa A, Pili M, Francomano D, et al. Effects of vardenafil administration on intravaginal ejaculatory latency in men with lifelong premature ejaculation. Int J Impot Res. 2009;21(4):221-227.

33. Mathers MJ, Klotz T, Roth S, Lummen G, Sommer F. Safety and efficacy of vardenafil versus sertraline in the treatment of premature ejaculation: a randomized, prospective and crossover study. Andrologia. 2009;41(3):169-175.

34. Salonia A, Maga T, Colombo R, et al. A prospective study comparing paroxetine alone versus paroxetine plus sildenafil in patients with premature ejaculation. $J$ Urol. 2002;168:2486-2489.

35. Chen J, Mabjeesh NJ, Matzkin H, Greenstein A. Efficacy of sildenafil as adjuvant therapy to selective serotonin reuptake inhibitors in alleviating premature ejaculation. Urology. 2003;61:197-200.

36. Wise ME, Watson JP. A new treatment for premature ejaculation: a case series for a de-sensitizing band. Sexual Relationship Therapy. 2000;15: 293-320.

37. Kim JJ, Kwak TI, Jeon BG, Cheon J, Moon DG. Effects of glans penis augmentation using hyaluronic acid gel for premature ejaculation. Int $J$ Impot Res. 2004;16:547-551.

38. Kwak TI, Jin MH, Kim JJ, Moon DG. Long term effects of glans penis augmentation using hyaluronic acid gel for premature ejaculation. Int $J$ Impot Res. 2008;20(4):425-428.

39. Basal S, Goktas S, Ergin A, et al. A novel treatment modality in patients with premature ejaculation resistant to conventional methods: the neuromodulation of dorsal penile nerves by pulsed radiofrequency. J Androl. 2009. Epub ahead of print.
Drug Design, Development and Therapy

\section{Publish your work in this journal}

Drug Design, Development and Therapy is an international, peerreviewed open-access journal that spans the spectrum of drug design and development through to clinical applications. Clinical outcomes, patient safety, and programs for the development and effective, safe, and sustained use of medicines are a feature of the journal,

\section{Dovepress}

which has also been accepted for indexing on PubMed Central. The manuscript management system is completely online and includes a very quick and fair peer-review system, which is all easy to use. Visit http://www.dovepress.com/testimonials.php to read real quotes from published authors. 